\title{
Overcoming Challenges in the Endoscopic Management of Sinonasal Tumors
}

\author{
${ }^{1}$ Vicky S Khattar, ${ }^{2}$ Bachi T Hathiram, ${ }^{3}$ Balagopal Kurup \\ ${ }^{1}$ Assistant Professor, Department of ENT and Head and Neck Surgery, TN Medical College and \\ BYL Nair Charitable Hospital, Mumbai, Maharashtra, India \\ ${ }^{2}$ Professor and Head, Department of ENT and Head and Neck Surgery, TN Medical College and \\ BYL Nair Charitable Hospital, Mumbai, Maharashtra, India \\ ${ }^{3}$ Resident, Department of ENT and Head and Neck Surgery, TN Medical College and \\ BYL Nair Charitable Hospital, Mumbai, Maharashtra, India
}

Correspondence: Vicky S Khattar, Assistant Professor, Department of ENT and Head and Neck Surgery, C-7, Swati Kalyan Complex, Panch Marg, Off Yari Road, Versova, Mumbai-400061, Maharashtra, India, Phone: 9930977110 e-mail: vickykhattar@ rediffmail.com, orlclinics@ gmail.com

\begin{abstract}
Introduction: The complex anatomical relationships of the sinonasal compartment with the surrounding vital structures make it vulnerable, yet simultaneously surgically accessible. Sinonasal tumors, especially those extending beyond, toward the anterior skull base and orbit have always posed a challenge, as far as their en bloc, and when applicable, oncologically safe removal is concerned. Traditionally, numerous external approaches have been employed for their successful removal. With advancements in endoscopic sinus surgical techniques and instrumentation, a majority of these tumors can be removed via purely transnasal endoscopic, and sometimes endoscopicassisted procedures

Materials and methods: A series of 61 sinonasal tumors have been managed by endoscopic or endoscopic-assisted procedures over the last 6 years. The principles of their management and the challenges posed have been highlighted. Methods to tackle the same, backed by evidence from numerous international studies have been applied to these, and the outcome discussed.

Conclusion: Endoscopic and endoscopic-assisted procedures are proving to be an effective alternative to the traditional external approaches for the management of sinonasal malignancies with comparable results. However, a long-term meta-analysis is imperative to corroborate these results.
\end{abstract}

Keywords: Neoplasms, Sinonasal tumors, Endoscope.

\section{INTRODUCTION}

The advent and successful outcomes of endoscopic sinus surgery have prompted surgeons to expand their horizons beyond the confines of the sinonasal compartment, toward the anterior skull base and orbit. Instruments and techniques, which were initially designed for inflammatory sinonasal pathologies, are today being increasingly applied to oncosurgery.

Endoscopic resection of sinonasal malignancy offers several advantages over the open approaches, including unparalled visualization that al lows precise tumor removal. It also allows for preservation of uninvolved structures, avoidance of facial incisions and superior cosmesis. ${ }^{1}$ In addition, there are practical advantages of an endonasal approach, such as the avoidance of an adverse functional impact on the nasal airflow, seen following the midfacial degloving or the lateral rhinotomy and medial maxillectomy approaches. ${ }^{2}$ This all in addition to the shorter hospital stay, lesser incidence of postoperative complications, faster rehabilitation into a normal lifestyle, etc.

$\mathrm{N}$ evertheless, there are many issues that need to be addressed. The inability of en bloc removal, cutting through tumor while piecemeal removal, width of margins, the appropriate adjuvant therapy to be employed, management of the orbit and the problem of recurrence. These issues have been discussed with respect to cases managed, and evidence-based techniques employed in an attempt to successfully overcome them.

\section{MATERIALS AND METHODS}

Over the past 6 years, 61 cases of sinonasal tumours have been managed (Table 1 ), including cases of hemangioma, angioma, juvenile angiofibroma, inverted papilloma, ossifying fibroma, neuroendocrine tumor, squamous cell carcinoma and sinonasal undifferentiated carcinoma. All patients underwent a standardized protocol for preoperative evaluation including a CT scan, diagnostic nasal endoscopic biopsy except in cases of angiomas, hemangiomas and juvenile angiofibromas. M RI scans were performed in all cases with suspected or CT scan-evident involvement of the orbit or skull base. Patients with vascular tumors underwent a digital subtraction angiography with embolization of the feeding vessel with polyvinyl alcohol particles, (and recently embosphere particles) 24 to 72 hours prior to surgery. All patients underwent an endonasal endoscopic excision of the tumor. In some cases, adjunctive exposure was achieved via a sublabial-transmaxillary approach (neuroendocrine with poorly differentiated 
squamous cell carcinoma), transcranial approach (squamous cell carcinoma) or a transoral approach (juvenile angiofibroma). In all cases, complete excision was possible. Depending on the histopathological diagnosis, and immunohistochemistry (when applicable), the patients were subjected to radiotherapy, and/or chemotherapy when required. All patients underwent endoscopic evaluation for 1 to 3 weeks postoperatively for removal of crusts. The first follow-up endoscopic evaluation was at 3 weeks after surgery, repeated every 3 months, with radiological evaluation at 6 months in cases of benign, and 3 months in cases of malignant tumors. Patients undergoing radiation therapy were scanned 6 months after completion of the course. There was two cases of a cerebrospinal fluid leak, two cases of recurrence in juvenile angiofibromas, two cases of recurrence in squamous cell carcinoma, two cases of recurrent neuroendocrine carcinomas, one case of inverting papilloma and one case of sinonasal undifferentiated carcinoma. Three of the squamous cell carcinomas were recurrent at presentation, and were only subjected to palliative treatment (debulking and radiotherapy). The followup period ranged from 17 to 49 months, and some patients are still on a follow-up. Certain cases required special attention and diversion from the above protocol, since they posed a challenge as regards diagnosis, treatment and postoperative adjunctive therapy, and have been discussed below. In these latter situations, consultation with oncosurgeons, ophthalmologists, neurosurgeons, radiation and medical oncologists and radiologists were undertaken. Similar or likewise situations mentioned in literature were also referred, so as to arrive at a customized management protocol for such difficult situations.

Table 1: The total number of cases managed

\begin{tabular}{llc}
\hline No. & Tumor & No. of cases \\
\hline 1 & Hemangioma & 4 \\
2 & Angioma & 5 \\
3 & Juvenile angiofibroma & 31 \\
4 & Ossifying fibroma & 1 \\
5 & Inverting papilloma & 5 \\
6 & Neuroendocrine tumor with & 1 \\
& coexisting squamous cell carcinoma & 1 \\
7 & Neuroendocrine carcinoma & \\
8 & Recurrent neuroendocrine carcinoma & 1 \\
9 & Rhinosporidiosis with coexisting & 5 \\
& squamous cell carcinoma & 3 \\
10 & Squamous cell carcinoma & \\
11 & Sinonasal undifferentiated carcinoma & 3 \\
12 & Recurrent squamous cell carcinoma & \\
& (at presentation) & \\
\hline
\end{tabular}

\section{DISCUSSION}

\section{Challenges}

With the switch-over from external to endoscopic approaches in the past two decades, numerous centers (including ours) encountered certain obstacles. These were initially more technical, but later dealt with tumor philosophy, tumor behavior and preservation of oncological principles. Once the necessary skill and expertize was present, wherein the clinician could confidentally handle such tumors endoscopically, numerous questions arose, as regard the safety profile of these procedures, with special reference to adherence to oncological principles. This led to further studies to eval uate these techniques and solutions were then put forward. A review of the constantly upgraded world literature on these techniques with correlation with our experience has been discussed.

\section{Infratemporal Fossa Involvement}

Even with the best angul ated endoscopes, the visual ization, and more so the access to the infratemporal fossa can be a daunting task for the endoscopic sinus surgeon. Commonly tumors like juvenile angiofibromas, have a tendency to grow into this space from the sphenopalatine foramen, via the pterygopalatine fossa. Endoscopic removal of the posterolateral wall of the maxillary sinus aids in releasing the bottlenecking of this tumor at the pterygopal atine fossa. Instruments and the endoscope can easily maneuver in the infratemporal fossa till a sagittal plane about $10 \mathrm{~mm}$ lateral to the lateral- most part of the maxillary sinus. For larger extensions, we were able to use a 'two-surgeon-four-hand technique' wherein the assisting surgeon gives traction to the otherwise completely dissected tumor from the opposite nostril via the choana with one hand, and the nasopharynx via the oral cavity with the other hand, both with angled Blakesley forceps. This has been likened to the removal of a retrosternal goiter from the upper mediastinum via a neck incision. The lateral limit of dissection is evident when one can see the infratemporal fossa fat protrude in the endoscopic surgical field.

In benign tumors, such as juvenile nasopharyngeal angiofibromas, which usually grow out from the pterygopalatine fossa to the infratemporal fossa, it is important to completely expose the lateral-most extent of the tumor prior to attempting removal. It may be prudent here to excise the medial wall of the maxilla, along with the inferior turbinate, so as to allow direct visualization of the tumor (Figs 1 and 2).

In fact, even with large tumors, this exposure offers excellent visualization, even with a zero degree sinuscope! Also, the moment the lateral-most extent of the tumor is reached, we have al most al ways come across a large vessel running vertically upward, just medial to the fat. This has been such a constant feature in our series of 31 angiofibromas, that we have actually used it as a benchmark to ensure complete lateral dissection of the tumor (Fig. 3). 


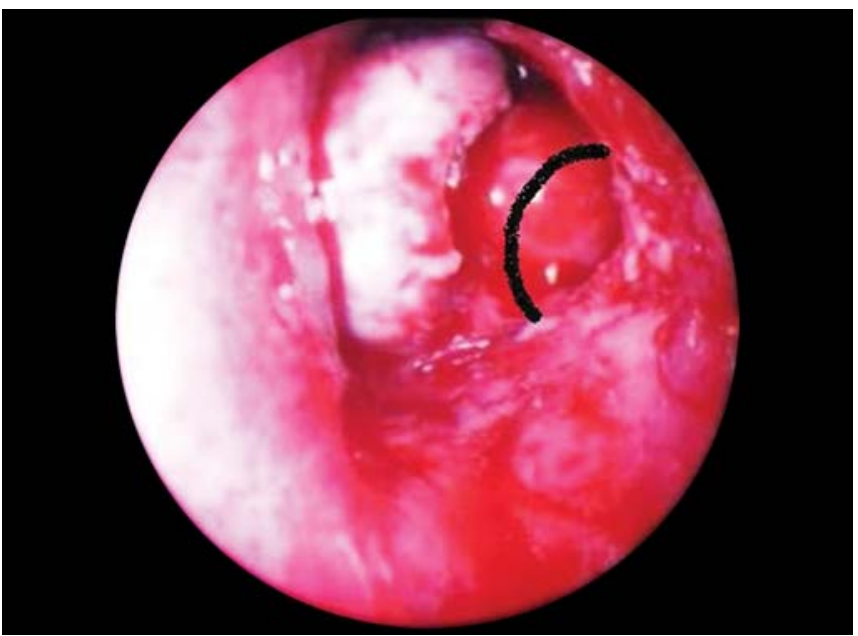

Fig. 1: Intraoperative endoscopic view of a left-sided juvenile nasopharyngeal angiofibroma with a large infratemporal fossa extension. The inferior turbinate (erstwhile position marked with the black line) and the medial maxillary wall have been removed to facilitate exposure of the entire tumor (seen)

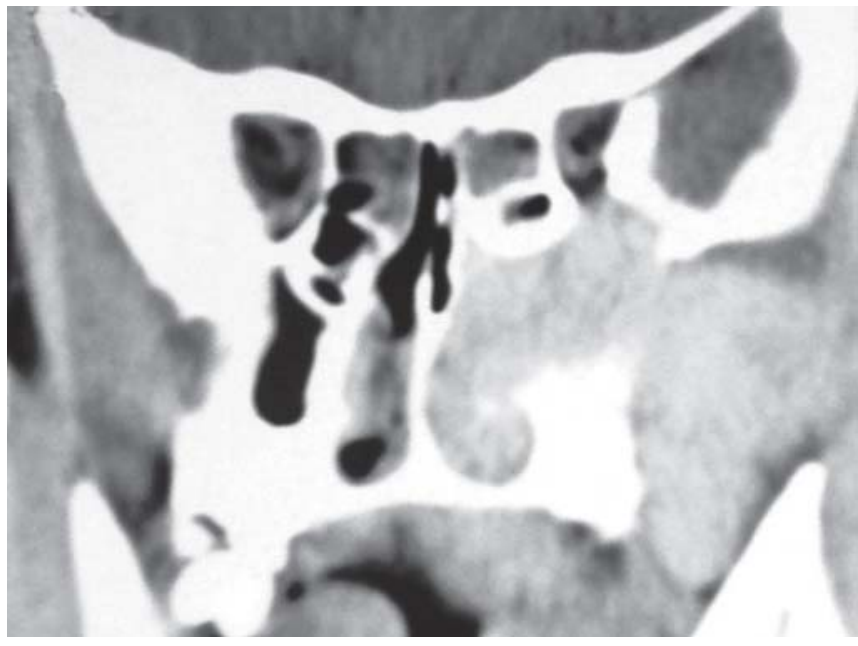

Fig. 2: The CT scan of the same tumor (Fig. 1)

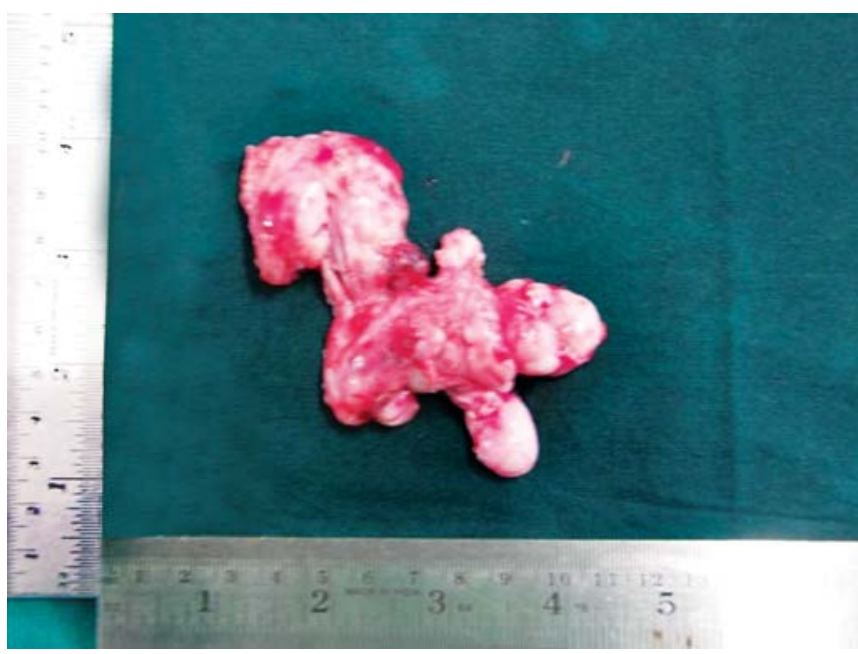

Fig. 3: Postoperative clinical photograph showing the entire specimen removed en bloc, showing its large infratemporal fossa component with its numerous 'pseudopods'

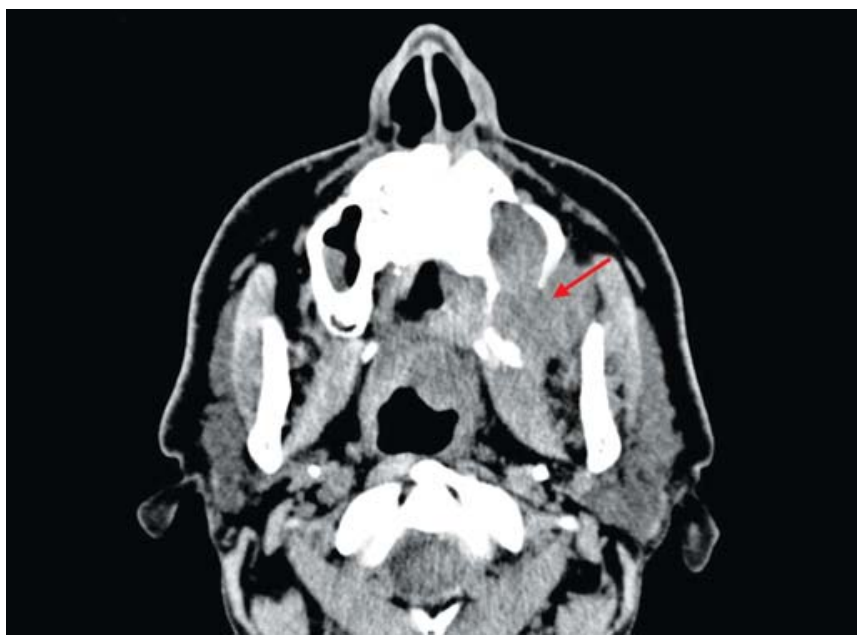

Fig. 4: Above axial CT scan showing the left infratemporal fossa component (red arrow) of the recurrent neuroendocrine tumor which was completely cleared

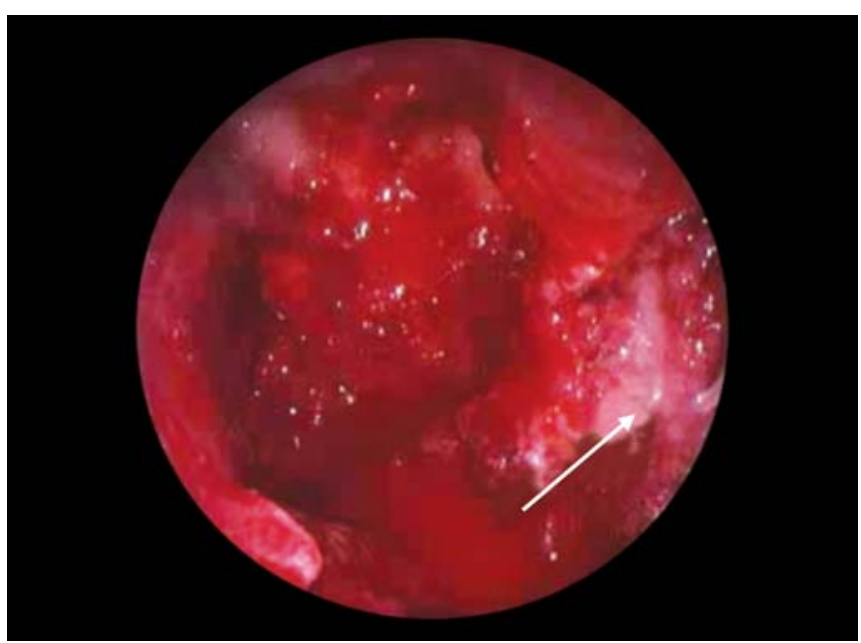

Fig. 5: The infratemporal fossa seen through the maxilla (white arrow) after removal of the tumor

In the case of a recurrent neuroendocrine tumor extending via the maxilla to the infratemporal fossa, along with erosion of the anterior and posterolateral maxillary wall, a combined endoscopic with sublabial-transmaxillary approach was used to access and remove the tumor (Fig. 4). Contrary to what could be seen on CT scan, the periosteum over the posterolateral wall of the maxilla was intact, but had been pushed into the infratemporal fossa by the tumor, and the tumor could thus be peeled off this periosteum (Fig. 5).

\section{Orbital Conservation}

Involvement of the orbit, especially in cases of malignancy can be a tricky affair. U sually in order to obtain oncologically safe margins, one would have to perform an orbital exenteration as the 'lateral resection margin'. 
Nevertheless, many authors have advocated a conservative approach, which includes conservative surgery, radiotherapy and regional chemotherapy as an effective method for local control and preservation of ocular function. ${ }^{3}$

Others have demonstrated a thin distinct fascial layer, which surrounds the periocular fat and separates it from the periorbita. ${ }^{4}$ Traditional literature proposed orbital exenteration for periorbita involvement, but studies, such as these ${ }^{4}$ have shown that the periosteum may be stripped off the underlying fat in an attempt to conserve the orbit.

This is in accordance with the concept that tumor removal in the sinonasal space and its periphery should be carried out for 'one compartment' beyond the tumor. By 'compartment' we would mean different things for different sites. For example, if the lamina papyracea is breached by a malignant tumor, it is removed along with the next compartment, namely the orbital periosteum. If the latter were to be involved, then the periosteum should be stripped off, leaving the fascial layer (vide supra) intact, and so on. in case of the ethmoids, if the anterior ethmoids were involved, then the posterior ethmoids and ground lamella would be removed behind, the middle turbinate medially, and the lamina papyracea laterally. Following this compartmentalized restriction of tumor margins, it was possible to salvage viable tissue, with removal of all macroscopically visible tumor, and confirming the remaining margins with frozen section. In fact, sometimes, we would have as many as 20 frozen section samples for a single case (Figs 6 to 8).

\section{Piecemeal vs en bloc Resection}

Rarely can a tumor be removed en bloc from the sinonasal space, by any approach, unless it is quite small. This inability, how ever, does not seem to have any impact on prognosis. ${ }^{5}$

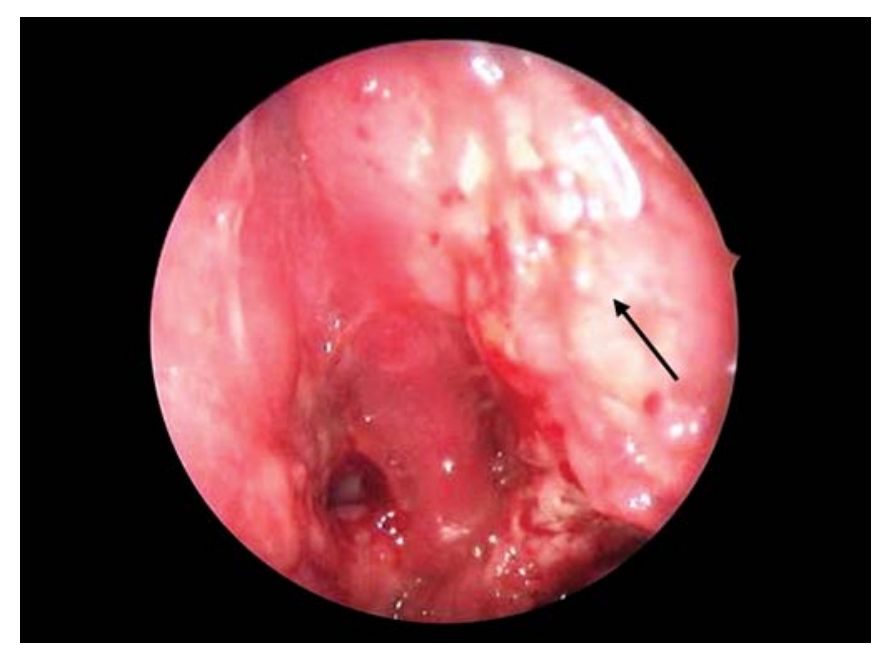

Fig. 6: The above postoperative endoscopic image shows healing mucosa over the orbital fat (black arrow) following stripping of the periosteum in a case of a recurrent squamous cell tumor in contact with the orbital periosteum as seen in figure 7 (red arrow)
In fact, the above authors have compared this with the transoral $\mathrm{CO}_{2}$ supraglottic laryngectomy in which cutting through the tumor also seems to have no impact on survival. In our experience, this holds true, and in this respect, sinonasal tumors have a different management protocol as compared to malignancies of the tongue, larynx, etc. where enblocremoval is the rule. A similar technique of piecemeal removal has been followed for Moh's micrographically controlled surgery for a long time.

However, a frozen section control of the margins is imperative, as discussed later.

$B$ atra and $\mathrm{Citardi}^{1}$ have described a minimally invasive endoscopic resection (MIER) technique as an alternative to traditional craniofacial resection, in which they have described two seemingly divergent surgical strategies. The first is the outside-in approach, in which the surgeon chooses to outflank the tumor by dissecting around the tumor, typically in a subperiosteal plane. W ehave used this technique more for juvenile angiofibromas and other benign tumors, since most malignant tumors are extremely friable. The other alternative is the inside-out approach, in which the core of large bulky malignant tumors is removed first, and the peripheral margins of the tumor are collapsed inward for removal. The other advantage of collapsing the periphery of malignant tumors inward was to allow for control of the blood vessels which commonly have been seen to supply the tumor from its periphery. In most instances, both strategies are used selectively in the same case. Our experience in cases of malignancies has been to commonly use a combination of both approaches for different components of the tumor (Figs 9 to 11).

Surprisingly, in the case of benign tumors such as juvenile angiofibromas, we have seen that a piecemeal removal allows for a higher chance of recurrence as compared to an en bloc removal. Thus, we strictly follow

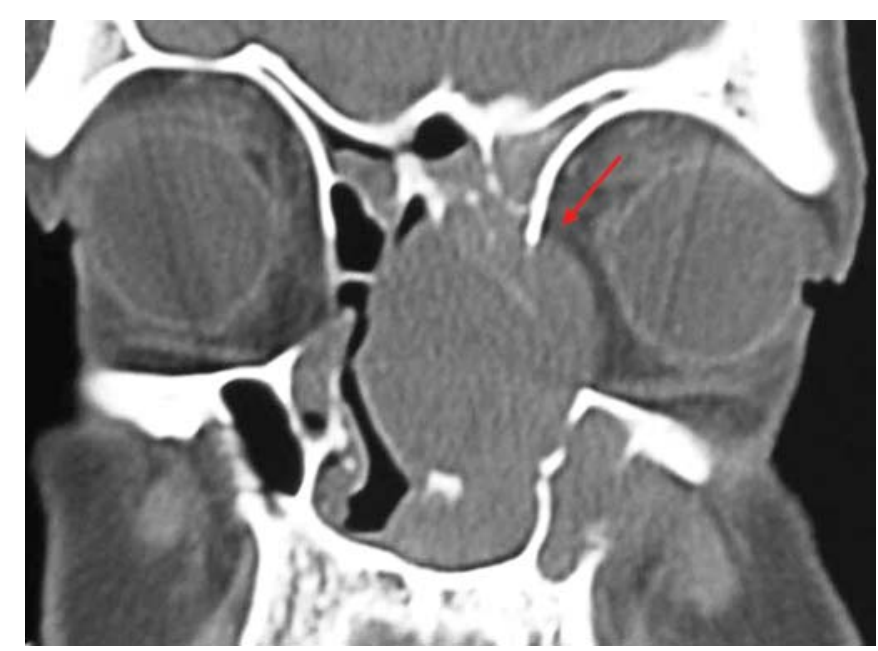

Fig. 7: Although in the CT scan of the patient in figure 6, the orbit looks invaded 


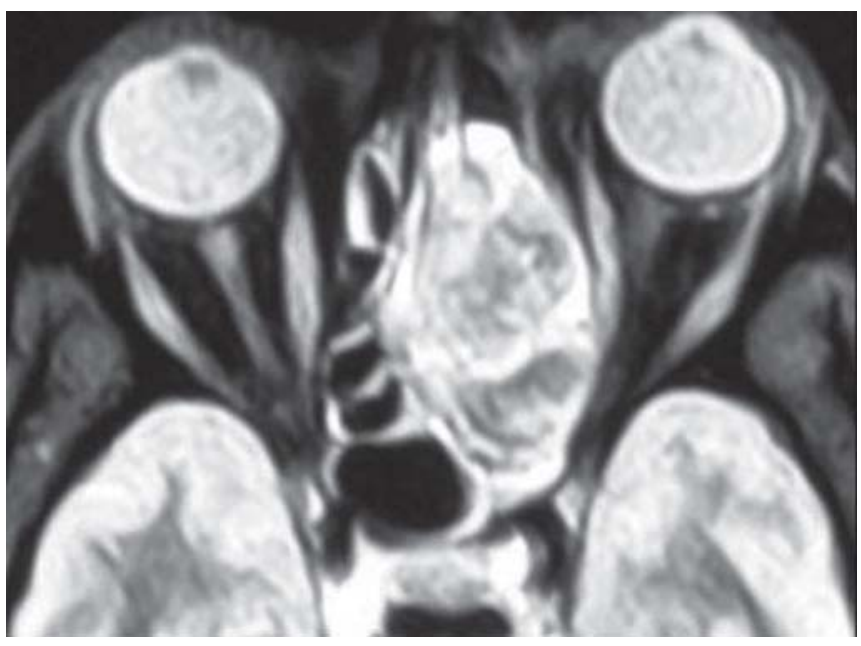

Fig. 8: The MRI scan of the same patient as in figures 6 and 7 reveals a displaced but uninvolved orbital periosteum which was confirmed intraoperatively and accordingly preserved

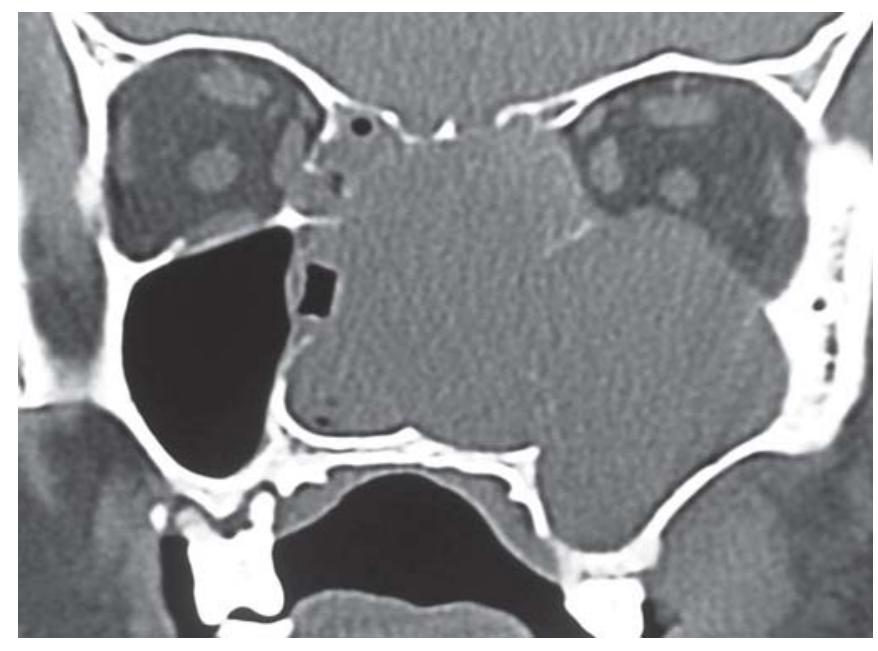

Fig. 9: The above coronal CT scan showing a large inverted papilloma which was removed by an inside-out technique as seen in Figure 10

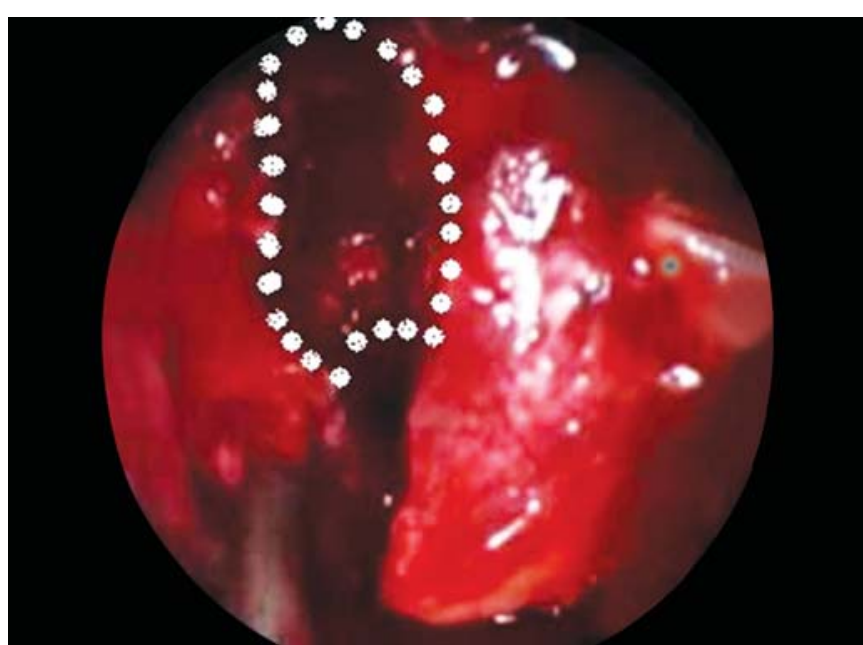

Fig. 10: The above intraoperative endoscopic image shows the same tumor being cored out (white dots) in order to debulk it

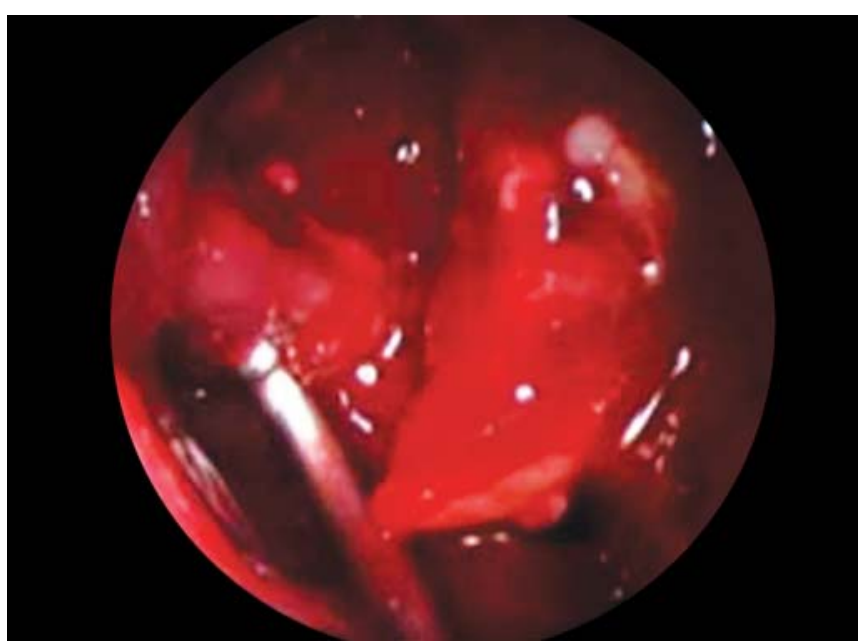

Fig. 11: The walls of the debulked tumor being gently peeled off the underlying tissues with a blunt probe. This allows for easy removal of very large tumors

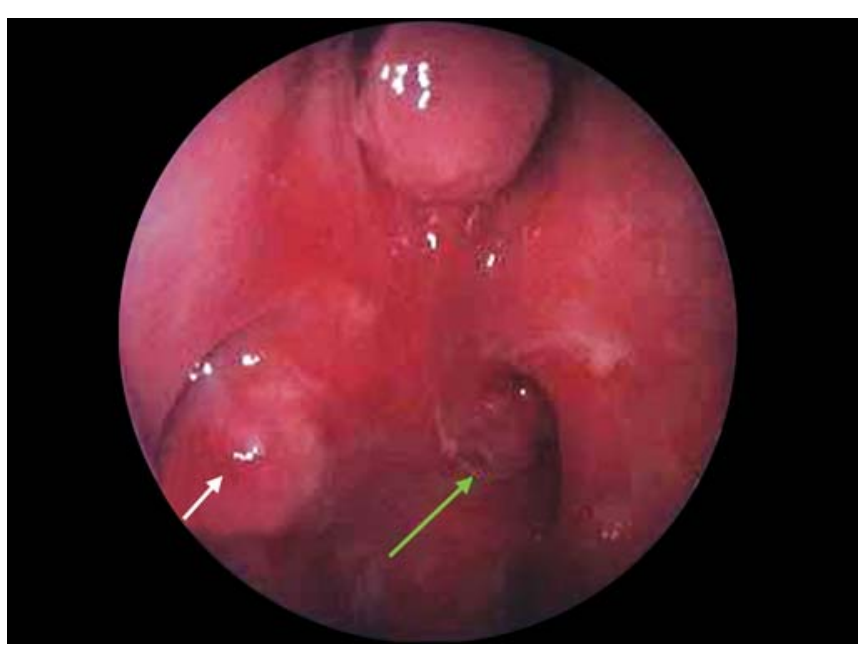

Fig. 12: The above 1-year postoperative endoscopic image shows a recurrence in the region of the right pterygopalatine fossa (white arrow) extending to the nasopharynx (green arrow) in case of a juvenile angiofibroma which broke during removal and had to be removed in a piecemeal fashion

the outside-in approach for juvenile angiofibromas (Fig. 12).

One exception was a case of a large nasopharyngeal angiofibroma, which was extending intracranially, with compression of the optic nerve and in contact with the dura. Once the sinonasal part of the tumor had been exposed and freed from its attachments, it remains attached to the intracranial extradural part. Dissection between the dura and the tumor would have to be carried out gently, with the risk of damaging the dura. W e thus, divided the tumor at its exit from the sphenoid sinus after cauterizing with a bipolar forceps, and then gently dissected off the remaining component. This allowed for minimal traction on the dura due to the lesser weight of the sphenoidal component, as compared to the entire tumor (Figs 13 to 17). 


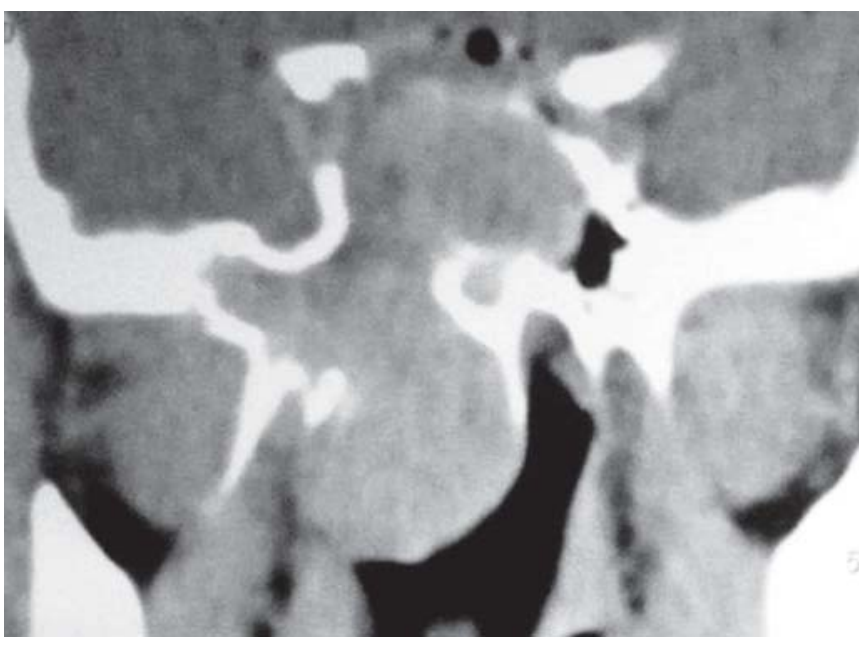

Fig. 13: Coronal enhanced CT scan of the paranasal sinuses showing the two 'components' of the tumor, i.e. nasal and sphenoidal (Fig. 14)

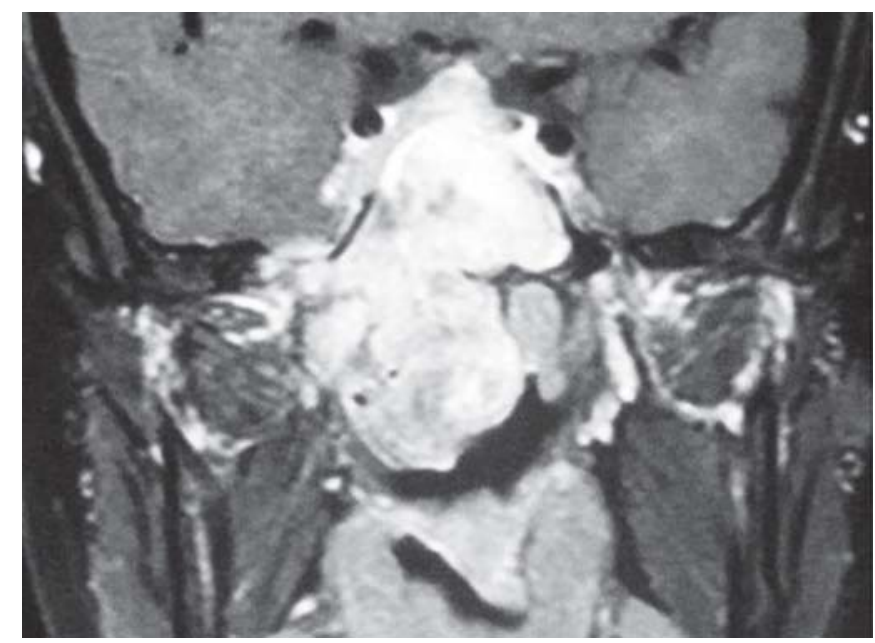

Fig. 14: Coronal MRI of the same patient (Fig. 13)

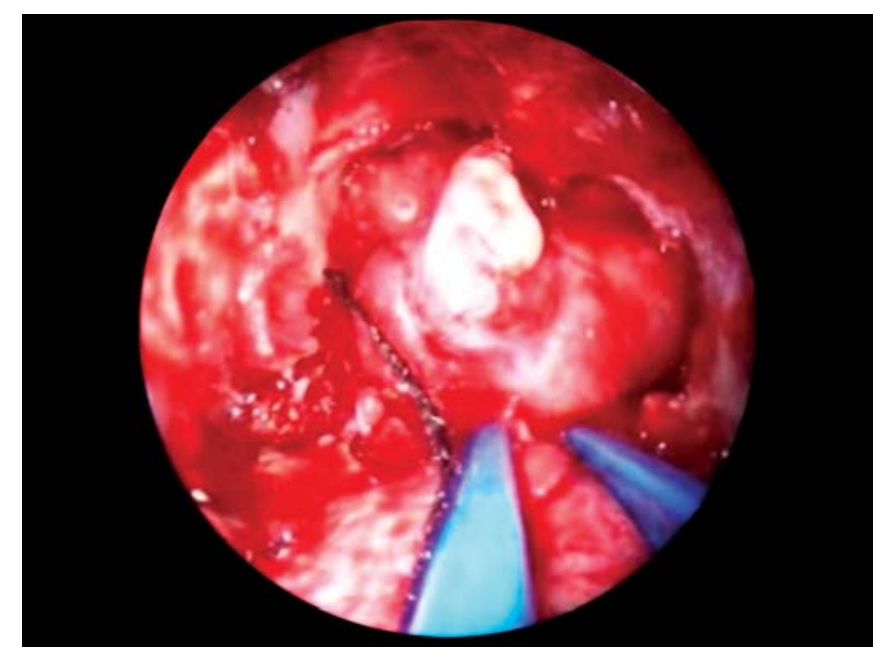

Fig. 15: A bipolar forceps being used to cauterize the tumor at the point where it emerges from the sphenoid sinus

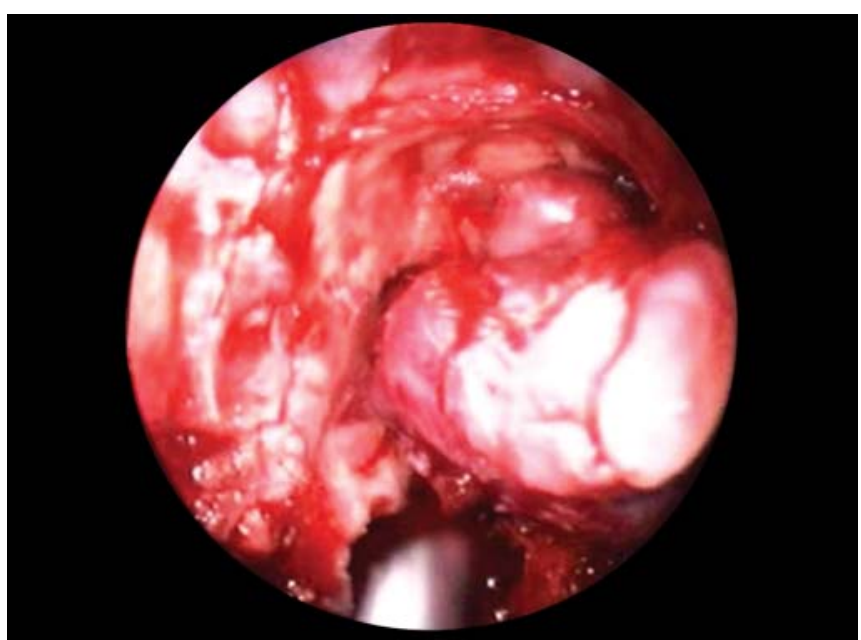

Fig. 16: Intraoperative endoscopic image showing the remnant 'sphenoidal' component of the tumor

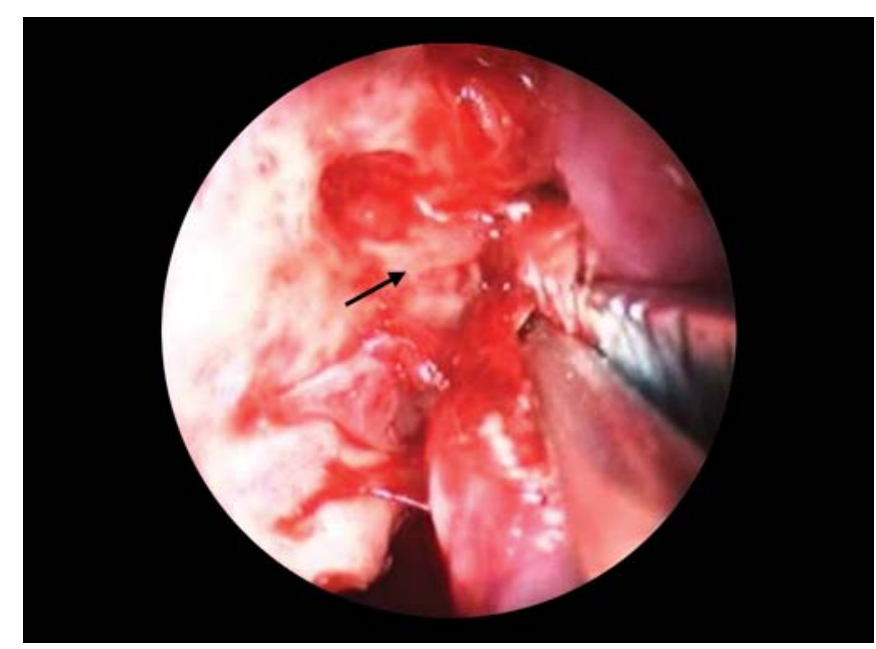

Fig. 17: The remnant component (Fig. 16) being gently peeled off the underlying optic nerve (black arrow) and dura above with minimal traction, due to its 'weight reduction' after dividing it initially

\section{Wide Margins}

Unlike other head and neck malignancies, where removal of the tumor with margins ranging from 1 to $5 \mathrm{~cm}$ is the rule, in the sinonasal compartment this is most of the times not possible. Similar to the orbit discussed above, the dura too can be respectively salvaged or resected. $M$ any malignant tumors are extradural, and a resection of the dura can be done in consultation with the neurosurgeons. Reconstruction is usually in two layers, with one placed transcranially (pericranial flap or fascia lata 'carpet graft') and the other transnasally endoscopically (fascialata). In tumors reaching just short of the dura, resection may be carried out till dura is reached.

A frozen section control of margins is however essential. This is more so since tumors are removed piecemeal. 
Once the bulk of the tumor has been removed, numerous biopsies are sent from various sites, and the same are mapped. Thus, for example, following removal of an ethmoid tumor, biopsies may be sent from the skull base (or dura) from numerous sites starting anteriorly, and moving posteriorly at every few millimeters intervals, with the same being mapped by an assistant diagrammatically, and numbered appropriately. A II the samples are sent in separate bottles for frozen section processing. If any biopsy turns positive, then those sites (and possibly the neighboring sites) are resected further till negative margins are achieved.

The same procedure may be done for the orbit (or periorbita), thenasal septum, etc. This mapping al so facilitates adjuvant postoperative therapy planning. Functional cavity concerns should be of secondary importance, and there should be no hesitation in removing all macroscopically involved tissue, be it dura, periorbita etc. (Harvey et al 2011). ${ }^{6}$ This holds true for malignant tumors; for benign tumors, such as inverted papillomas, preserving anatomic barriers to spread is of paramount importance (Figs 18 and 19).

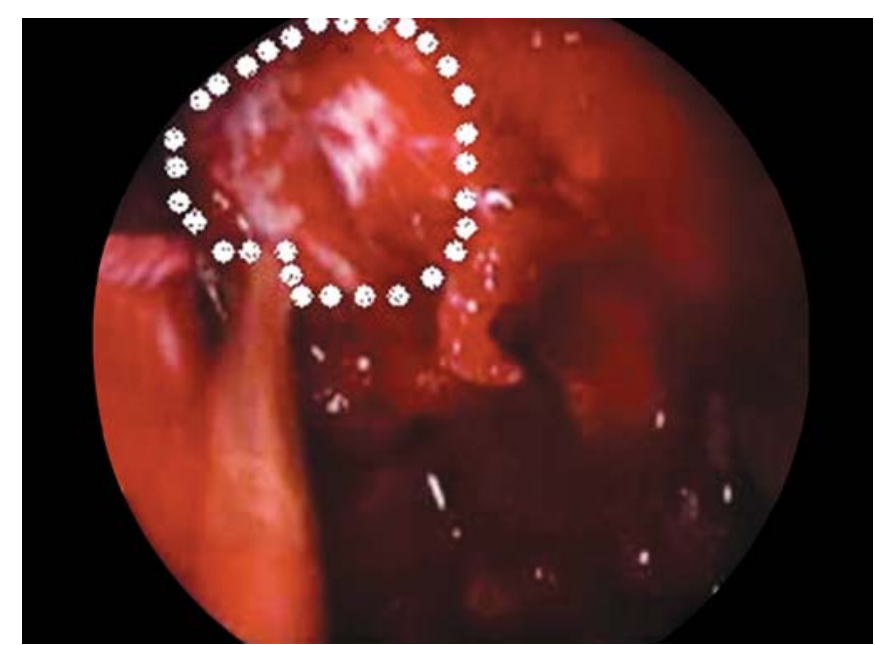

Fig. 18: The white dots in the above intraoperative endoscopic image show an intact and clinically normal appearing dura following resection of an esthesioneuroblastoma extending

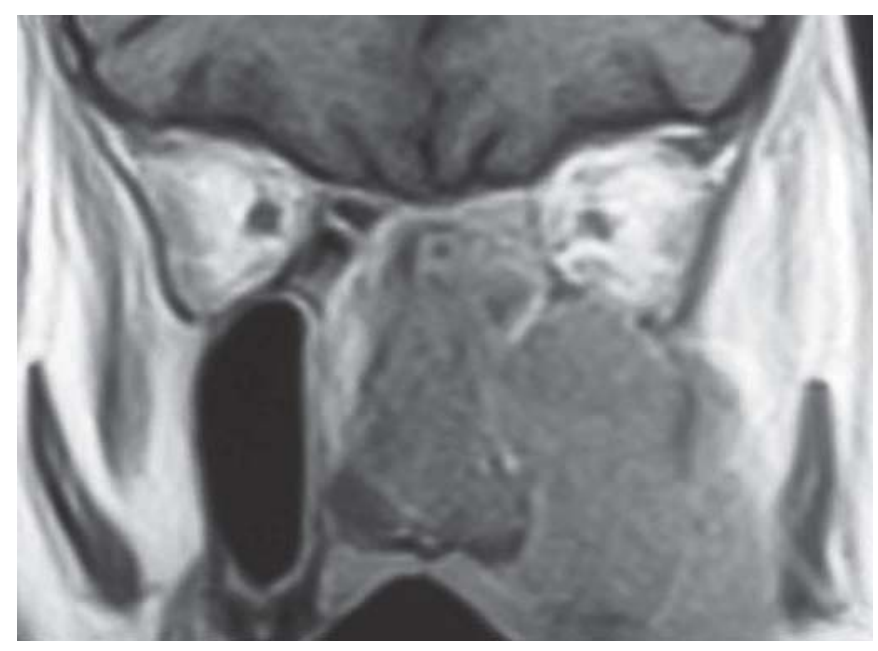

Fig. 19: J ust short of the dura as seen from the MRI (Fig. 18)

\section{Palliation vs Cure}

The surgical objectives of complete tumor removal must be balanced with the goal of minimizing adverse impact on the 'quality of life' of patients (Batra et al 2010). ${ }^{7}$ M any patients suffering from malignant tumors are incurable right from the time of presentation. In considering palliative treatment, it is necessary to record the patient's symptoms, and appreciate how they impact on their life, the extent of their disease, and whether distant metastasis is present (M CM onagle and Gleeson 2008). It is imperative here to take the patient and their relatives into confidence, and explain the expected outcome of the treatment. They may then be subjected to a palliative debulking, with postoperative radiotherapy. O ften, repeated debulking may be performed at varying time intervals.

\section{Preoperative vs Postoperative Radiotherapy}

In radiobiological terms, preoperative radiotherapy has traditionally been advocated; with modern techniques, this seldom interferes with healing (M CM onagle and Gleeson ${ }^{8}$ 2008), and also carries with it the added advantage of tumor shrinkage, better definition of tumor margins, etc. In slow growing tumors, such as adenoid cystic carcinomas and chondrosarcomas, postoperative radiotherapy may have a more valuable role (M cM onagle and Gleeson 2008). In malignant tumors with intracranial extension, preoperative radiotherapy, with planned surgery (endoscopic-assisted craniofacial resection) after 6 weeks is now accepted by many centers as a treatment modality.

\section{The Role of Chemotherapy}

A majority of sinonasal tumors belong to the squamous cell and adenocarcinoma variety, for which chemotherapy has little to offer. Chemotherapy is hel pful in managing olfactory neuroblastoma, Iymphoma, rhabdomyosarcoma and sinonasal undifferentiated carcinoma (M CM onagle and Gleeson 2008). Kengt et $\mathrm{al}^{9}$ have advocated repeated necrotomy and application of topical 5-fluorouracil for adenocarcinoma of the ethmoids, and this is being practiced by some centers.

\section{RESULTS}

In this series, among the benign tumors, there were two recurrences, both in juvenile angiofibromas. A mong the malignant tumors, there were two cases of recurrence in squamous cell carcinoma, two cases of recurrent neuroendocrine carcinomas, one case of inverting papilloma and one case of sinonasal undifferentiated carcinoma. The case of neuroendocrine carcinoma with coexisting poorly differentiated carcinoma has only been on a 7 months follow-up, and will require a longer period of time to depict 
the overall prognosis. The tumors that had been operated with a palliative intent, were subjected to adjunctive radiotherapy (if the option had not al ready been exhausted as in one case).

\section{CONCLUSION}

The sample size in the above series and in most other mentioned in literature so far are small, and hence statistical significance may not be present. A long with larger series, a multicentric meta-analysis with standardized protocols may help in determining the success or failure of this technique. Having said that with modifications in technique, yet maintaining oncological principles, endoscopic resection of sinonasal tumors may prove to be an effective alternative to the traditional external approaches. One must keep in mind though that whatever the approach used, a multi-disciplinary approach with a team of dedicated clinicians is imperative for a successful outcome of this pathology, which otherwise can have a highly morbid outcome oncologically, functionally and cosmetically.

\section{ACKNOWLEDG MENT}

The authors would like to thank the Dean, TN Medical College and BY L N air Charitable H ospital, M umbai, India for permission to publish this article.

\section{REFERENCES}

1. Batra PS, Citardi MJ. Endoscopic management of sinonasal malignancy. Otolaryngol Clin N A m 2006;39:619-37.

2. Lueg $E A$, I rish JC, Roth $Y$, et al. A n objective analysis of the impact of lateral rhinotomy and medial maxillectomy on nasal airway function. L aryngoscope 1998;108:1320-24.

3. Sato Y, M orita M , Takahashi H, W atanabe N, K irikael. Combined surgery, radiotherapy, and regional chemotherapy in carcinoma of the paranasal sinuses. Cancer 1983;52:1360-64.

4. Tiwari R, van der Wal J, van der W aal I, Snow G. Studies of the anatomy and pathology of the orbit in carcinoma of the maxillary sinus and their impact on preservation of the eye in maxillectomy. Head Neck 1998;20(3):193-96.

5. Rice D. Endonasal approaches for sinonasal and nasopharyngeal tumours. Otolaryngol Clin N A m 2001;34:1087-93.

6. Harvey RJ, Winder M, Parmar P, Lund V. Endoscopic skull base surgery for sinonasal malignancy. Otolaryngol Clin N A m 2011;44:1081-1140.

7. Batra PS, Luong A, K anowitz SJ, Sade B, Lee J, Lanza DC, Citardi MJ. Outcomes of minimally invasive endoscopic resection of anterior skull base neoplasms. Laryngoscope 2010;120:9-16.

8. M cM onagle BA, Gleeson M . Nasal cavity and paranasal sinus malignancy. Scott B rown's otorhinolaryngology, head and neck surgery (7th edn), Edward Arnold (publishers) Ltd., London, UK 2008;2:2417-36.

9. Knegt PP, Ah-See KW, vd V elden LA, Kerrebjin J. A denocarcinoma of the ethmoidal sinus complex: Surgical debulking and topical fluouracil may be the optimal reatment. A rch Otolaryngol Head Neck Surg 2001;127:141-46. 